\title{
ON SOME REGULARITY PROPERTIES FOR SOLUTIONS OF NONLINEAR PARABOLIC DIFFERENTIAL EQUATIONS
}

\author{
HARUO NAGASE
}

\section{Introduction}

Let $G$ be a bounded domain in $R^{n}$ with coordinates $x=\left(x_{1}, \ldots, x_{n}\right)$ and let its boundary $S$ be of class $C^{2}$. We assume that the usual function spaces $L^{q}(G)$, $W^{1, q}(G)$ and $W_{0}^{1, q}(G), 1<q<\infty$, are known. We write the norm of $L^{q}(G)$ by $\mid l_{q}$ and the adjoint number of $q$ by $q^{*}$, i.e., $q^{*}=q /(q-1)$.

For any positive number $T$ we denote the open interval $(0, T)$ by $I$, the cylinder $G \times I$ in $R^{n+1}$ by $Q$ and the norm of $L^{q}(Q)$ by \|\|$_{q}$.

Let $X$ be a Banach space with the norm ||$_{X}$. Let $C(I, X)$ be the space of all continuous functions $v(t) ; I \rightarrow X$. Let us denote by $L^{q}(I, X), 1<q<\infty$, and $L^{\infty}(I, X)$ the set of all measurable functions $v(t) ; I \rightarrow X$ such that $|v|_{L^{q(I, X)}}$ $=\left(\int_{I}|v(t)|_{X}^{q} d t\right)^{1 / q}<\infty$ and $|v|_{L^{\infty}(I, X)}=\underset{I}{\operatorname{ess} \sup }|v(t)|_{X}<\infty$, respectively. A function $v(t) ; I \rightarrow X$ is said to be strongly differentiable at $t \in I$, if there exists $w(t)$ in $X$ such that $\lim |(u(t+h)-u(t)) / h-w(t)|_{X}=0$. Then we call $w(t)$ by the strong derivative of $v(t)$ at $t \in I$ and we denote it by $v_{t}(t)$.

In this paper we make the assumption

$$
2(n+1) /(n+3)<p<2 .
$$

Let us denote $W_{0}^{1, p}(G)$ by $V$, its dual space by $V^{*}$, the pairing between $V$ and $V^{*}$ by $\langle$,$\rangle and the inner product of L^{2}(G)$ by $($,$) . Further, we write$ $\mathbf{0}=(0, \ldots, 0) \in R^{n}$ and $D v=\left(v_{x_{j}}\right), j=1, \ldots, n, v_{x_{j}}=\left(\partial / \partial x_{j}\right) v$.

We define a nonlinear operator $A(t) ; V \rightarrow V^{*}, t \in I$, as follows:

$$
\langle A(t) v, w\rangle=\sum_{j=1}^{n}\left(a_{j}(., t, D v), w_{x_{j}}\right), v, w \in V .
$$

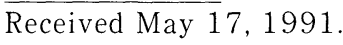


Let us suppose that functions $a_{j}(x, t, \eta), j=1, \ldots, n$, satisfy the assumption

$$
\begin{aligned}
& a_{\jmath}(x, t, \eta) \in C^{0}\left(Q \times R^{n}\right) \cap C^{1}\left(Q \times\left(R^{n}-\{\mathbf{0}\}\right)\right), \\
& a_{j}(x, t, \mathbf{0})=0, j=1, \ldots, n,
\end{aligned}
$$

and for $(x, t) \in Q, \eta \in R^{n}-\{\mathbf{0}\}, \xi \in R^{n}$,

$$
\begin{aligned}
& \sum_{i, j=1}^{n}\left(\partial / \partial \eta_{i}\right) a_{j}(x, t, \eta) \xi_{\imath} \xi_{j} \geqq \gamma|\eta|^{p-2}|\xi|^{2}, \\
& \left|\left(\partial / \partial \eta_{\imath}\right) a_{j}(x, t, \eta)\right| \leqq \Lambda|\eta|^{p-2}, i, j=1, \ldots, n \\
& \left|\left(\partial / \partial x_{k}\right) a_{j}(x, t, \eta)\right|,\left|(\partial / \partial t) a_{j}(x, t, \eta)\right| \leqq \Lambda|\eta|^{p-1}, j, k=1, \ldots, n .
\end{aligned}
$$

Here $\gamma$ and $\Lambda$ are some positive constants.

Now we consider the nonlinear parabolic differential equation

(2) $\left\{\begin{array}{l}u \in L^{\infty}(I, V) \cap C\left(I, L^{2}(G)\right), u_{t} \in L^{2}\left(I, L^{2}(G)\right), \\ \left(u_{t}(t), \phi\right)+\langle A(t) u(t), \phi\rangle=\langle f(t), \phi\rangle \text { for any } \phi \in V \text { and a.e. } t \in I, \\ u(x, 0)=u_{0}(x),\end{array}\right.$

In this paper we suppose that $f$ and $u_{0}$ in (2) satisfy the following assumptions (A.3) and (A.4) respectively:

(A.3) $f \in L^{p *}\left(I, W^{1, p^{*}}(G)\right)$ and $f_{t} \in L^{2}\left(I, L^{2}(G)\right)$.

(A.4) $u_{0} \in V$ and $D u_{0} \in L^{2}(G)$. Further, there exists a function $z_{0}$ in $L^{2}(G)$ such that the equality

$$
\left(z_{0}, v\right)+\left\langle A(0) u_{0}, v\right\rangle=\langle f(0), v\rangle
$$

holds for all $v \in V$, where $f(0)=f(x, 0) \in V^{*}$.

Recently in [7] we showed the unique existence of solutions such that $u_{t} \in$ $L^{\infty}\left(I, L^{2}(G)\right)$ and gave some a priori estimates for solutions of nonlinear parabolic variational inequalities, which contain the equation (2) in a special case, when $2 \leqq p$. We can prove the unique existence of solutions of (2) by the similar way as in [7] when the assumption (A.1) is satisfied also. The aim of this paper is to show some regularity properties of solutions of (2) in such cases.

Before stating our results we introduce some notations

$$
\begin{aligned}
& k(s)=2 s /(s+2-p), m(s)=2 s /(s+4-2 p) \text { and } \\
& p_{\infty}=2(n+1)(p-1) /(n-1) .
\end{aligned}
$$


Remark 1. It is easy to see that the followings hold:

(i) If $p \leqq s<p_{\infty}$, then $k(s) \leqq s$ and $p \leqq k(s)<k\left(p_{\infty}\right)$.

(ii) If $p<2$, then $m(s)<k(s)<2$.

Our theorem is as follows:

THEOREM. Under the assumptions (A.1)-(A.4) it holds that for the unique solution $u$ of (2)

(i) $D^{2} u \in L^{k\left(p_{\infty}\right)}\left(I^{\prime}, L_{\mathrm{loc}}^{k\left(p_{\infty}\right)}(G)\right),(D u)_{t} \in L^{k\left(p_{\infty}\right)}\left(G \times I^{\prime}\right)$,

(ii) $D^{2} u \in L^{m\left(p_{\infty}\right)}\left(G \times I^{\prime}\right)$,

where $D^{2} u=\left(u_{x_{i} x}\right),(D u)_{t}=\left(u_{x_{j} t}\right), 1 \leqq i, j \leqq n, I^{\prime}=(a, b)$ and $0<a<$ $b<T$.

Remark 2. It is easy to see that $k\left(p_{\infty}\right)=4(n+1)(p-1) /((n+3) p-4)$ $>p$ and $m\left(p_{\infty}\right)=2(n+1)(p-1) /(2 p+n-3)$. Further, $k\left(p_{\infty}\right) \rightarrow 2$ and $m\left(p_{\infty}\right) \rightarrow 2$ as $p \rightarrow 2$.

S. Campanato [3], F. de Thelin [4], J. Naumann [8] and others showed the interior integrability of the second order derivatives of solutions of nonlinear elliptic differential equations and systems. Moreover, J.P. Raymond [9] proved the global integrability of the second order derivatives of solutions of nonlinear elliptic differential systems. N.D. Alikakos-L.C. Evans [1], N.D. Alikakos-R. Rostamian [2], E. DiBenedetto-A. Friedman [5] and others obtained some estimates and some integrability properties of the first order derivatives of solutions of nonlinear parabolic differential equations. But we have few results with respect to the integrability of the second order derivatives of solutions of nonlinear parabolic differential equations.

We attempt to extend the results in [9] to solutions of the nonlinear parabolic differential equation (2).

This paper is constructed as follows. In Section 1 we prepare some preliminary lemmas without proofs. In Section 2 we show some lemmas which play important roles in the proof of our theorem. Proofs of these lemmas are given by the method of the difference quotient. The proof of our theorem is given in Section 4 .

1.

Lemma1. Under the assumption (A.2) there exists a positive constant $\gamma_{0}$ such that 


$$
\begin{aligned}
& \sum_{j=1}^{n}\left(a_{j}(x, t, \eta)-a_{j}\left(x, t, \eta^{\prime}\right)\right)\left(\eta_{j}-\eta_{j}^{\prime}\right) \\
\geqq & \gamma_{0}\left(|\eta|+\left|\eta^{\prime}\right|\right)^{p-2}\left|\eta-\eta^{\prime}\right|^{2}
\end{aligned}
$$

where $(x, t) \in Q, \eta, \eta^{\prime} \in R^{n}$ and $|\eta|+\left|\eta^{\prime}\right| \neq 0$.

The above lemma was proved by P. Tolksdorf in [11, p.246, Lemma 2.3].

Let $v$ be a function in a bounded domain $\Omega$ in $R^{m}$ and let us denote $e_{\imath}=(0, \ldots, 0, \dot{1}, 0, \ldots, 0), 1 \leqq i \leqq m$. We define the difference quotient by

$$
\delta_{i}^{h} v(x)=\left(v\left(x+h e_{\imath}\right)-v(x)\right) / h, h \neq 0 .
$$

The following lemma plays an important role in this paper:

Lemma 2 [6, p.168, Lemmas 7.23, 7.24]. (i) Let $v \in W^{1, q}(\Omega), 1<q<\infty$. Then for any $\Omega^{\prime} \subset \bar{\Omega} \subset \Omega$ and $|h|<\operatorname{dis}\left(\Omega^{\prime}, \partial \Omega\right)$ the estimate

$$
\left|\delta_{i}^{h} v\right|_{L^{q}\left(\Omega^{\prime}\right)} \leqq C\left|v_{x_{i}}\right|_{L^{q}(\Omega)}
$$

holds, where $\partial \Omega$ is the boundary of $\Omega$ and $C$ is a positive constant which does not depend on $v$ and $h$.

(ii) Let $v \in L^{q}(\Omega)$ and let the inequality $\left|\delta_{\imath}^{h} v\right|_{L^{q\left(\Omega^{\prime}\right)}} \leqq C$ be valid for any $h$ and $\Omega^{\prime} \subset \bar{\Omega} \subset \Omega$ such that $|h|<\operatorname{dis}\left(\Omega^{\prime}, \partial \Omega\right)$. Then $v_{x_{i}} \in L^{q}\left(\Omega^{\prime}\right)$ and

$$
\left|v_{x_{i}}\right|_{L^{q}\left(\Omega^{\prime}\right)} \leqq C
$$

Next, let us prepare the reverse Hölder's inequality, see [10, p.8].

Lemma 3. Let $0<r<1$ and $r^{*}=r /(r-1)$. If $F \in L^{r}(\Omega), F H \in L^{1}(\Omega)$ and $\int_{\Omega}|H(x)|^{r^{*}} d x<\infty$, then it holds that

$$
\left(\int_{\Omega}|F(x)|^{r} d x\right)^{1 / r} \leqq\left(\int_{\Omega}|F(x) H(x)| d x\right)\left(\int_{\Omega}|H(x)|^{r^{*}} d x\right)^{-1 / r^{*}}
$$

Here $\Omega$ is a bounded domain in $R^{m}$.

2.

In this section we denote any sufficiently small open set in $R^{n}$ by $U$ and $U^{\prime}$. Let us assume that $U \subset U^{\prime}$ and $U \cap S \neq \phi$. We see that there exists a diffeomorphism $g$; $R^{n} \rightarrow R^{n}$ which satisfies the followings: 


$$
\begin{aligned}
& g(U)=B_{r}, g\left(U^{\prime}\right)=B_{R}, 0<r<R, \\
& g(G \cap U)=B_{r} \cap\left\{x_{n}>0\right\}, g\left(G \cap U^{\prime}\right)=B_{R} \cap\left\{x_{n}>0\right\},
\end{aligned}
$$

where $B_{\rho}=\left\{x \in R^{n} ;|x|<\rho\right\}$. From now on we consider the problem only in the case that

(2.1) $G \cap U=B_{r} \cap\left\{x_{n}>0\right\}, G \cap U^{\prime}=B_{R} \cap\left\{x_{n}>0\right\}, 0<r<R$, in order to simplify calculations. We can show all of results in this paper by calculations with minor changes in the general case, see [9].

We denote $x=\left(x^{\prime}, x_{n}\right), x^{\prime}=\left(x_{1}, \ldots, x_{n-1}\right)$ and $D^{\prime} v=\left(v_{x_{j}}\right), 1 \leqq j \leqq n-1$.

In this section let us suppose that the assumptions (A.1)-(A.4) are satisfied. And let us denote any solution of (2) by $u$.

Hereafter, we write by the same $C$ all constants which do not depend on $u$, $u_{0}, z_{0}$ and $f$.

Lemma 4. If $U \cap S \neq \phi$, then

$$
\sup _{I}\left|D^{\prime} u(t)\right|_{L^{2}(G \cap U)} \leqq M
$$

and $D^{\prime}(D u) \in L^{p}((G \cap U) \times I)$ with $D^{\prime}(D u)=\left(u_{x_{i} x_{j}}\right),(i, j) \neq(n, n), 1 \leqq$ $\mathrm{i}, j \leqq n$. Here $M$ is a positive constant depending on $\left|D u_{0}\right|_{2},\|D f\|_{p^{*}}$ and $\|D u\|_{p}$.

Proof. Let $\zeta(x)$ be a $C_{0}^{\infty}\left(B_{R}\right)$-function such that $0 \leqq \zeta \leqq 1$ and $\zeta=1$ on $B_{r}$. For any sufficiently small $|h|$ we denote $\Delta_{i}^{h} v=\Delta_{i}^{h} v(x, t)=v\left(x+h e_{i}, t\right)$ $-v(x, t), i \neq n$. Taking $\phi=\Delta_{i}^{-h}\left(\zeta \Delta_{i}^{h} u\right)$ as a test function in (2), we have

$$
\begin{aligned}
& \left(u_{t}(t), \Delta_{i}^{-h}\left(\zeta \Delta_{i}^{h} u\right)(t)\right)+\left\langle A(t) u(t), \Delta_{i}^{-h}\left(\zeta \Delta_{i}^{h} u\right)(t)\right\rangle \\
= & \left\langle f(t), \Delta_{\imath}^{-h}\left(\zeta \Delta_{i}^{h} u\right)(t)\right\rangle .
\end{aligned}
$$

Each term on the left-hand side of (2.3) is rewritten as follows:

$$
\left.\begin{array}{rl} 
& \left(u_{t}(t), \Delta_{i}^{-h}\left(\zeta \Delta_{i}^{h} u\right)(t)\right)=\left(\Delta_{i}^{h} u_{t}(t), \zeta \Delta_{i}^{h} u(t)\right) \\
= & (1 / 2)(d / d t)\left|\zeta^{1 / 2} \Delta_{i}^{h} u(t)\right|_{2}^{2},
\end{array}\right\}
$$

From (2.3)-(2.5) 


$$
\begin{aligned}
(1 / 2)(d / d t)\left|\zeta^{1 / 2} \Delta_{i}^{h} u(t)\right|_{2}^{2}+\sum_{j=1}^{n}\left(\Delta_{i}^{h} a_{j}(., t, D u), \zeta \Delta_{i}^{h} u_{x_{j}}(t)\right) \\
=\left\langle\Delta_{\imath}^{h} f(t), \zeta \Delta_{\imath}^{h} u(t)\right\rangle-\sum_{j=1}^{n}\left(a_{j}(., t, D u), \Delta_{\imath}^{-h}\left(\zeta_{x_{j}} \Delta_{\imath}^{h} u\right)(t)\right)
\end{aligned}
$$

For any $t \in I$ let us integrate (2.6) over $(0, t)$. Then

$$
\begin{aligned}
& (1 / 2)\left|\zeta^{1 / 2} \Delta_{\imath}^{h} u(t)\right|_{2}^{2}+\sum_{j=1}^{n}\left[\Delta_{i}^{h} a,(., ., D u), \zeta \Delta_{i}^{h} u_{x j}\right]_{Q t} \\
& =(1 / 2)\left|\zeta^{1 / 2} \Delta_{i}^{h} u(0)\right|_{2}^{2}+\left[\Delta_{\imath}^{h} f, \zeta \Delta_{\imath}^{h} u\right]_{Q t} \\
& -\sum_{j=1}^{n}\left[a_{j}(., ., D u), \Delta_{i}^{-h}\left(\zeta_{x}, \Delta_{\imath}^{h} u\right)\right]_{Q t} .
\end{aligned}
$$

Here we have denoted the inner product of $L^{2}\left(Q_{t}\right)$ by $[,]_{Q t}$, where $Q_{t}=Q_{(0, t)}$ $=G \times(0, t)$.

The second term on the left-hand side of (2.7) is rewritten in the form:

$$
\begin{aligned}
& \quad \sum_{j=1}^{n}\left[\Delta_{i}^{h} a_{j}(., ., D u), \zeta \Delta_{i}^{h} u_{x_{j}}\right]_{Q t}=\sum_{j=1}^{n}\left[a_{j}\left(x+h e_{i}, ., D u\left(x+h e_{i}, .\right)\right)\right. \\
& \left.-a_{j}\left(x+h e_{i}, ., D u(x, .)\right), \zeta\left(u_{x_{j}}\left(x+h e_{\imath}, .\right)-u_{x_{j}}(x, .)\right)\right]_{Q t} \\
& +\sum_{j=1}^{n}\left[a_{j}\left(x+h e_{i}, ., D u(x, .)\right)-a_{j}(x, ., D u(x, .)), \zeta\left(u_{x_{j}}\left(x+h e_{i}, .\right)\right.\right. \\
& \left.\left.-u_{x}(x, .)\right)\right]_{Q t} .
\end{aligned}
$$

Each term on the right-hand side of (2.8) is estimated as follows:

$$
\begin{aligned}
& \text { the first term } \geqq \gamma_{0} \int_{Q t}\left(|D u|+\left|D u_{h, i}\right|\right)^{p-2}\left|D u_{h, i}-D u\right|^{2} \zeta d z \text {, } \\
& \leqq C|h| \int_{Q t}\left(|D u|+\left|D u_{h, i}\right|\right)^{p-1}\left|D u_{h, i}-D u\right| \zeta d z \\
& \leqq\left(\gamma_{0} / 2\right) \int_{Q t}\left(|D u|+\left|D u_{h, i}\right|\right)^{p-2}\left|D u_{h, i}-D u\right|^{2} \zeta d z \\
& +C h^{2} \int_{Q t}|D u|^{p} d z
\end{aligned}
$$

where $d z=d x d t, u_{h, t}=u\left(x+h e_{l}, t\right)$ and we have defined the integrand $\left(|D u|+\left|D u_{h, i}\right|\right)^{p-2}\left|D u_{h, i}-D u\right|^{2}$ to be zero on $\left\{(x, t) \in Q_{t} ;|D u|=\right.$ $\left.\left|D u_{h, i}\right|=0\right\}$. In the above we have used Lemma 1, the assumption (A.2) and Young's inequality. 
Using Lemma 2, Hölder's inequality and the assumption (A.2) again, each term on the right-hand side of (2.7) is estimated as follows:

$$
\begin{aligned}
& (1 / 2)\left|\zeta^{1 / 2} \Delta_{\imath}^{h} u(0)\right|_{2}^{2} \leqq C h^{2}\left|D u_{0}\right|_{2}^{2}, \\
& \left|\left[\Delta_{i}^{h} f, \zeta \Delta_{i}^{h} u\right]_{Q_{l}}\right| \leqq C h^{2}\|D f\|_{p^{*}}\|D u\|_{p}, \\
& \left|\sum_{j=1}^{n}\left[a_{j}(., ., D u), \Delta_{i}^{-h}\left(\zeta_{x_{j}} \Delta_{\imath}^{h} u\right)\right]_{Q^{\prime}}\right| \\
& \leqq \sum_{j=1}^{n}\left\|a_{j}(., ., D u)\right\|_{p^{*}}\left|\Delta_{i}^{-h}\left(\zeta_{x,} \Delta_{i}^{h} u\right)\right|_{L^{p}\left(Q_{t}\right)} \\
& \leqq C|h|\|D u\|_{p}^{p-1} \sum_{j=1}^{n}\left(\left\|\zeta_{x_{i x j}} \Delta_{\imath}^{h} u\right\|_{p}+\left|\zeta_{x_{j}} \Delta_{i}^{h} D u\right|_{L^{p}\left(Q_{t}\right)}\right) \\
& \leqq C h^{2}\|D u\|_{p}^{p}+C|h|\|D u\|_{p}^{p-1}\left|\zeta_{x_{j}} \Delta_{i}^{h} D u\right|_{L^{p}\left(Q_{t}\right)} \\
& \leqq C h^{2}\|D u\|_{p}^{p}+C|h|\|D u\|_{p}^{p-1}\left|\zeta^{1 / p} \Delta_{i}^{h} D u\right|_{L^{p}\left(Q_{t}\right)},
\end{aligned}
$$

because we can choose the function $\zeta$ such that $\left|\zeta_{x_{j}}\right|^{p} \leqq C \zeta$.

Accordingly, from (2.7)-(2.13) we deduce the inequality

$$
\begin{aligned}
& (1 / 2)\left|\zeta^{1 / 2} \Delta_{\imath}^{h} u(t)\right|_{2}^{2} \\
& \quad+\left(\gamma_{0} / 2\right) \int_{Q t}\left(|D u|+\left|D u_{h, i}\right|\right)^{p-2}\left|D u_{h, 2}-D u\right|^{2} \zeta d z \\
\leqq & C h^{2}\left(\|D u\|_{p}^{p}+\left|D u_{0}\right|_{2}^{2}+\|D f\|_{p *}^{p *}\right)+C|h|\|D u\|_{p}^{p-1}\left|\zeta^{1 / p} \Delta_{i}^{h} D u\right|_{L^{p}(Q t)} .
\end{aligned}
$$

Next setting $r=p / 2, F=\zeta^{2 / p}\left|\Delta_{i}^{h} D u\right|^{2}, H=\left(|D u|+\left|D u_{h, l}\right|\right)^{p-2} \zeta^{(1-2 / p)}$, $\Omega=\hat{Q}_{t}$ and $\hat{Q}_{t}=\left\{(x, t) \in Q_{t} ; \zeta\left(|D u|+\left|D u_{h, l}\right|\right) \neq 0\right\}$, let us use Lemma 3 . Then

$$
\begin{aligned}
\text { (2.15) } & \left(\int_{\hat{Q}_{t}}\left|\Delta_{i}^{h} D u\right|^{p} \zeta d z\right)^{2 / p}=\left(\int_{\hat{Q}_{t}}\left(\left|\Delta_{i}^{h} D u\right|^{2} \zeta^{2 / p}\right)^{p / 2} d z\right)^{2 / p} \\
\leqq & \left(\int_{\widehat{Q}_{t}}\left(|D u|+\left|D u_{h, i}\right|\right)^{p-2} \zeta^{(1-2 / p)}\left|\Delta_{i}^{h} D u\right|^{2} \zeta^{2 / p} d z\right) . \\
& \left(\int_{\hat{Q}_{t}}\left\{\left(|D u|+\left|D u_{h, i}\right|\right)^{p-2} \zeta^{(1-2 / p)}\right\}^{(p / 2) *} d z\right)^{-1 /(p / 2) *} \\
= & \left(\int_{Q_{t}}\left(|D u|+\left|D u_{h, i}\right|\right)^{p-2}\left|\Delta_{i}^{h} D u\right|^{2} \zeta d z\right)\left(\int_{Q_{t}}\left(|D u|+\left|D u_{h, i}\right|\right)^{p} \zeta\right.
\end{aligned}
$$

In the above we have used the relations $(p-2)(p / 2)^{*}=p$ and $(1-2 / p)$ $(p / 2)^{*}=1$. It is easy to see that the estimate $(2.15)$ is valid if we replace $\hat{Q}_{t}$ by $Q_{t}$. Consequently, from (2.14) and (2.15) 


$$
\left(\int_{Q t}\left|\Delta_{i}^{h} D u\right|^{p} \zeta d z\right)^{2 / p} \leqq C_{1} h^{2}+C_{2}|h|\left(\int_{Q t}\left|\Delta_{\imath}^{h} D u\right|^{2} \zeta d z\right)^{1 / p} .
$$

From now on we denote by $C_{i}, i=1, \ldots$, any positive constant which depends on $u_{0}, f$ and $u$ only.

By Young's inequality again

$$
\left(\int_{Q t}\left|\Delta_{i}^{h} D u\right|^{p} \zeta d z\right)^{2 / p} \leqq C_{3} h^{2}
$$

Furthermore, by virtue of (2.14) and (2.17) we obtain the estimate

$$
\left|\Delta_{i}^{h} u(t) \zeta^{1 / 2}\right|_{2}^{2} \leqq C_{4} h^{2} \text { for any } t \in I .
$$

Hence we arrive at (2.2) from Lemma 2 and (2.18).

Next, after setting $t=T$ in (2.17), let us Lemma 2 again. Then we see that $D^{\prime}(D u) \in L^{p}((G \cap U) \times I)$.

Remark 3. If $U \cap S=\phi$, then $\sup _{I}|D u(t)|_{L^{2}(G \cap U)} \leqq M$ and $D^{2} u \in$ $L^{p}((G \cap U) \times I)$.

Lemma 5. Let $D u \in L^{s}(G \times J)$ with $s, p \leqq s \leqq p_{\infty}$, and $J=(c, d), 0 \leqq$ $c<d \leqq T$. If $U \cap S \neq \phi$, then $D^{\prime}(D u) \in L^{k(s)}((G \cap U) \times J)$ and $(D u)_{t} \in$ $L^{k(s)}\left(G \times J^{\prime}\right)$ for any $J^{\prime}=\left(c^{\prime}, d^{\prime}\right), c<c^{\prime}<d^{\prime}<d$.

The proof of this lemma is similar to that of Lemma 4. However, for the completeness we give it.

Proof. At first let us integrate (2.6) over $J$. Then, denoting the inner product of $L^{2}\left(Q_{J}\right)$ by $[,]_{Q_{J}}, Q_{J}=G \times J$, we obtain the equality

$$
\begin{aligned}
& (1 / 2)\left|\zeta^{1 / 2} \Delta_{i}^{h} u(d)\right|_{2}^{2}+\sum_{j=1}^{n}\left[\Delta_{i}^{h} a_{j}(., ., D u), \zeta \Delta_{i}^{h} u_{x_{j}}\right]_{Q J} \\
= & (1 / 2)\left|\zeta^{1 / 2} \Delta_{i}^{h} u(c)\right|_{2}^{2}+\left[\Delta_{i}^{h} f, \zeta \Delta_{i}^{h} u\right]_{Q J} \\
& -\sum_{j=1}^{n}\left[a,(., ., D u), \Delta_{i}^{-h}\left(\zeta_{x_{j}} \Delta_{i}^{h} u\right)\right]_{Q J} .
\end{aligned}
$$

By the same way as in (2.8)-(2.10) we have the inequality

$$
\sum_{j=1}^{n}\left[\Delta_{i}^{h} a_{j}(., ., D u), \zeta \Delta_{i}^{h} u_{x J}\right]_{Q J}
$$




$$
\geqq\left(\gamma_{0} / 2\right) \int_{Q_{J}}\left(|D u|+\left|D u_{h, i}\right|\right)^{p-2}\left|D u_{h, i}-D u\right|^{2} \zeta d z-C h^{2}\|D u\|_{p}^{p}
$$

Next, from (2.18)

$$
\left|\zeta^{1 / 2} \Delta_{\imath}^{h} u(c)\right|_{2}^{2} \leqq C_{4} h^{2}
$$

Further, by (2.13)

$$
\begin{aligned}
& \left|\sum_{j=1}^{n}\left[a_{j}(., ., D u), \Delta_{\imath}^{-h}\left(\zeta_{x_{j}} \Delta_{\imath}^{h} u\right)\right]_{Q_{J}}\right| \\
& \leqq C h^{2}\|D u\|_{p}^{p}+C|h|\|D u\|_{p}^{p-1}\left|\zeta_{x_{j}} \Delta_{\imath}^{h} D u\right|_{L^{p}\left(Q_{J}\right)} .
\end{aligned}
$$

Let us choose the function $\zeta$ such that $\left|\zeta_{x_{j}}\right|^{k(s)} \leqq C \zeta$ and let us remark the inequality $p \leqq k(s)$, see Remark 1 . Then, from (2.22)

$$
\begin{aligned}
& \left|\sum_{j=1}^{n}\left[a_{j}(., ., D u), \Delta_{i}^{-h}\left(\zeta_{x_{j}} \Delta_{i}^{h} u\right)\right]_{Q_{J}}\right| \\
\leqq & C h^{2}\|D u\|_{p}^{p}+C|h|\|D u\|_{p}^{p-1}\left|\zeta^{1 / k(s)} \Delta_{i}^{h} D u\right|_{L^{k(s)}\left(Q_{J}\right)} .
\end{aligned}
$$

Combining (2.19)-(2.21), (2.23) with (2.12), we see that

$$
\begin{aligned}
& \int_{Q J}\left(|D u|+\left|D u_{h, 2}\right|\right)^{p-2}\left|D u_{h, 2}-D u\right|^{2} \zeta d z \\
\leqq & C h^{2}\left(\|D u\|_{p}^{p}+C_{4}+\|D f\|_{p^{*}}^{p^{*}}\right) \\
& +C|h|\|D u\|_{p}^{p-1}\left|\zeta^{1 / k(s)} \Delta_{i}^{h} D u\right|_{L^{k(s)}\left(Q_{J}\right)} .
\end{aligned}
$$

Setting $r=k(s) / 2, F=\zeta^{2 / k(s)}\left|\Delta_{i}^{h} D u\right|^{2}, H=\left(|D u|+\left|D u_{h, \imath}\right|\right)^{p-2} \zeta^{(1-2 / k(s))}$, $\Omega=\hat{Q}_{J}$ and $\hat{Q}_{J}=\left\{(x, t) \in Q_{J} ; \zeta\left(|D u|+\left|D u_{h, i}\right|\right) \neq 0\right\}$, let us use Lemma 3 again. Then

$$
\begin{aligned}
& \left(\int_{\hat{Q}_{J}}\left|\Delta_{i}^{h} D u\right|^{k(s)} \zeta d z\right)^{2 / k(s)}=\left(\int_{\hat{Q}_{J}}\left(\left|\Delta_{\imath}^{h} D u\right|^{2} \zeta^{2 / k(s)}\right)^{k(s) / 2} d z\right)^{2 / k(s)} \\
\leqq & \left(\int_{\hat{Q}_{J}}\left(|D u|+\left|D u_{h, i}\right|\right)^{p-2} \zeta^{(1-2 / k(s))}\left|\Delta_{i}^{h} D u\right|^{2} \zeta^{2 / k(s)} d z\right) . \\
& \left(\int_{\hat{Q}_{J}}\left\{\left(|D u|+\left|D u_{h, i}\right|\right)^{p-2} \zeta^{(1-2 / k(s))}\right\}^{(k(s) / 2) *} d z\right)^{-1 /(k(s) / 2) *} \\
= & \left(\int_{Q_{J}}\left(|D u|+\left|D u_{h, i}\right|\right)^{p-2}\left|\Delta_{i}^{h} D u\right|^{2} \zeta d z\right) . \\
& \left(\int_{Q_{J}}\left(|D u|+\left|D u_{h, i}\right|\right)^{s} \zeta d z\right)^{(2-p) / s} .
\end{aligned}
$$

In the above we have used the relations $(p-2)(k(s) / 2)^{*}=s$ and $(1-2 / k(s))$. 
$(k(s) / 2)^{*}=1$. It is easy to see that the estimate (2.25) is valid also if we replace $\hat{Q}_{J}$ by $Q_{J}$.

From (2.24) and (2.25)

$$
\begin{aligned}
& \left(\int_{Q_{J}}\left|\Delta_{i}^{h} D u\right|^{k(s)} \zeta d z\right)^{2 / k(s)} \\
& \leqq\left(C_{5} h^{2}+C_{6}|h|\left|\zeta^{1 / k(s)} \Delta_{j}^{h} D u\right|_{L^{k(s)}\left(Q_{J}\right)}\right)\left(\int_{Q_{J}}|D u|^{\mathrm{s}} \zeta d z\right)^{(2-p) / s}
\end{aligned}
$$

By Young's inequality again

$$
\left(\int_{Q J}\left|\Delta_{l}^{h} D u\right|^{k(s)} \zeta d z\right)^{2 / k(s)} \leqq h^{2}\left(C_{7}\left|\zeta^{1 / s} D u\right|_{L^{s}\left(Q_{J}\right)}^{2-p_{j}}+C_{8}\left|\zeta^{1 / s} D u\right|_{\left.L^{(S(Q)}\right)}^{2\left(2-p_{j}\right)}\right)
$$

Using Lemma 2, we finish the proof for the first part of this lemma.

Next we show the second part of this lemma. Its proof is similar to those of Lemma 4 and the first part of this lemma. Let $\xi(t)$ be a $C_{0}^{\infty}(I)$-function such that $0 \leqq \xi \leqq 1, \xi=1$ on $J^{\prime}$ and $\operatorname{supp} \xi=J$. For any sufficiently small $|h|$ we denote $\Delta_{t}^{h} v=\Delta_{t}^{h} v(x, t)=v(x, t+h)-v(x, t)$. Taking $\phi=\Delta_{t}^{-h}\left(\xi \Delta_{t}^{h} u\right)$ as a test function in (2), we have

$$
\begin{aligned}
& \left(u_{t}(t), \Delta_{t}^{-h}\left(\xi \Delta_{t}^{h} u\right)(t)\right)+\left\langle A(t) u(t), \Delta_{t}^{-h}\left(\xi \Delta_{t}^{h} u\right)(t)\right\rangle \\
= & \left\langle f(t), \Delta_{t}^{-h}\left(\xi \Delta_{t}^{h} u\right)(t)\right\rangle .
\end{aligned}
$$

Let us integrate (2.28) over $I$. Then

$$
\begin{aligned}
& {\left[u_{t}, \Delta_{t}^{-h}\left(\xi \Delta_{t}^{h} u\right)\right]+\int_{I}\left\langle A(t) u(t), \Delta_{t}^{-h}\left(\xi \Delta_{t}^{h} u\right)(t)\right\rangle d t } \\
= & {\left[f, \Delta_{t}^{-h}\left(\xi \Delta_{t}^{h} u\right)\right], }
\end{aligned}
$$

where we have denoted the inner product in $L^{2}(Q)$ by [ . ].

By simple calculations

$$
\begin{aligned}
& {\left[u_{t}, \Delta_{t}^{-h}\left(\xi \Delta_{t}^{h} u\right)\right] } \\
= & {\left[\Delta_{t}^{h} u_{t}, \xi \Delta_{t}^{h} u\right]=(1 / 2)\left\{\int_{0}^{T}(d / d t)\left|\xi^{1 / 2} \Delta_{t}^{h} u\right|_{2}^{2} d t-\left[\Delta_{t}^{h} u, \xi^{\prime} \Delta_{t}^{h} u\right]\right\} } \\
= & (1 / 2)\left\{\left[\left|\xi^{1 / 2} \Delta_{t}^{h} u\right|_{2}^{2}\right]_{t=0}^{t=T}-\left[\Delta_{t}^{h} u, \xi^{\prime} \Delta_{t}^{h} u\right]\right\} \\
= & -(1 / 2)\left[\Delta_{t}^{h} u, \xi^{\prime} \Delta_{t}^{h} u\right],
\end{aligned}
$$

because $\xi(0)=\xi(T)=0$. Therefore, from Lemma 2

$$
\left|\left[u_{t}, \Delta_{t}^{-h}\left(\xi \Delta_{t}^{h} u\right)\right]\right| \leqq C h^{2}\left\|u_{t}\right\|_{2}^{2} .
$$

We rewrite the second term on the left-hand side of (2.29) into the form 


$$
\begin{aligned}
& \int_{I}\left\langle A(t) u(t), \Delta_{t}^{-h}\left(\xi \Delta_{t}^{h} u\right)(t)\right\rangle d t=\sum_{j=1}^{n}\left[a_{\jmath}(., ., D u),\left(\Delta_{t}^{-h}\left(\xi \Delta_{t}^{h} u\right)\right)_{x_{j}}\right] \\
= & \sum_{j=1}^{n}\left[\Delta_{t}^{h} a_{1}(., ., D u), \xi \Delta_{t}^{h} u_{x_{j}}\right] .
\end{aligned}
$$

By the same way as in (2.8)-(2.10) the right-hand side of (2.31) is estimated from below as follows:

$$
\begin{aligned}
& \sum_{j=1}^{n}\left[\Delta_{t}^{h} a_{j}(., ., D u), \xi \Delta_{t}^{h} u_{x j}\right] \\
\geqq & \left(\gamma_{0} / 2\right) \int_{Q}\left(|D u|+\left|D u_{h, t}\right|\right)^{p-2}\left|D u_{h, t}-D u\right|^{2} \xi d z-C h^{2}\|D u\|_{p}^{p},
\end{aligned}
$$

where $u_{h, t}=u(x, t+h)$.

Moreover, the right-hand side of (2.29) is estimated from Lemma 2. Namely,

$$
\left|\left[f, \Delta_{t}^{-h}\left(\xi \Delta_{t}^{h} u\right)\right]\right|=\left|\left[\Delta_{t}^{h} f, \xi \Delta_{t}^{h} u\right]\right| \leqq C h^{2}\left\|f_{t}\right\|_{2}\left\|u_{t}\right\|_{2} .
$$

From (2.29)-(2.33) it yields that

$$
\int_{Q}\left(\left|D u_{h, t}\right|+|D u|\right)^{p-2}\left|D u_{h, t}-D u\right|^{2} \xi d z \leqq C_{9} h^{2} .
$$

Employing the same calculations as in (2.25), we arrive at the following estimate from $(2.34)$ :

$$
\left(\int_{Q}\left|\Delta_{t}^{h} D u\right|^{k(s)} \xi d z\right)^{2 / k(s)} \leqq C_{10} h^{2}\left\|\xi^{1 / s} D u\right\|_{s}^{2-p} .
$$

By Lemma 2 we finish the proof of this lemma.

q.e.d.

Remark 4. Suppose that the same conditions as in Lemma 5 are satisfied. If $U \cap S=\phi$, then $D^{2} u \in L^{h(s)}((G \cap U) \times J)$. This is shown by the same way as in the above proof.

Lemia 6. Let $D u \in L^{s}(G \times J)$ with $s, p \leqq s \leqq p_{\infty}$, and $J=(c, d), 0 \leqq c$ $<d \leqq T$. If $U \cap S \neq \phi$, then $D^{2} u \in L^{m(s)}((G \cap U) \times J)$.

Proof. It holds that $u_{x_{i} x_{j}} \in L^{k(s)}((G \cap U) \times J)$ for any $(i, j) \neq(n, n)$ from Lemma 5 and $m(s)<k(s)$ from Remark 1 . Then let us show that $u_{x_{n} x_{n}} \in L^{m(s)}$ $((G \cap U) \times J)$ only. 
By virtue of Lemma 5 and Nikodym's theorem the following equality holds in the usual sense a.e. $Q$ :

$$
\begin{aligned}
& \left(\partial / \partial \eta_{n}\right) a_{n}(x, t, D u) u_{x_{n} x_{n}}=u_{t}(x, t)-f(x, t) \\
& \quad-\sum_{j=1}^{n}\left(\partial / \partial x_{j}\right) a_{j}(x, t, D u)-\sum_{(i, j) \neq(n, n)}\left(\partial / \partial \eta_{\imath}\right) a_{j}(x, t, D u) u_{x_{i} x_{j}} .
\end{aligned}
$$

It is valid that $u_{x_{n} x_{n}}=0$ a.e. $N$ with $N=\{(x, t) \in Q ;|D u|=0\}$. Hence it is enough for us to estimate $u_{x_{n} x_{n}}$ on $Q \backslash N$. After multiplying (2.36) by $u_{x_{n} x_{n}}$, let us use the assumption (A.2). Then

$$
\begin{aligned}
|D u|^{p-2}\left|u_{x_{n} x_{n}}\right|^{2} \leqq C\left|u_{x_{n} x_{n}}\right| & \left(\left|u_{t}\right|+|f|+\sum_{j=1}^{n}\left|\left(\partial / \partial x_{j}\right) a_{j}\right|\right. \\
& \left.+\sum_{(i, j) \neq(n, n)}\left|\left(\partial / \partial \eta_{i}\right) a_{j}\right|\left|u_{x_{i} x_{j}}\right|\right) \text { a.e. } Q \backslash N .
\end{aligned}
$$

Therefore, using the assumption (A.2) again, we have

$$
\left|u_{x_{n} x_{n}}\right| \leqq C\left(|D u|^{2-p}\left|u_{t}\right|+|D u|^{2-p}|f|+|D u|+\sum_{(i, j) \neq(n, n)}\left|u_{x_{i} x_{j}}\right|\right)
$$

It is evident that (2.37) holds a.e. $N$ also. Let us consider each term on the right-hand side of (2.37). If follows that both $|D u|^{2-p}\left|u_{t}\right|$ and $|D u|^{2-p}$. $|f|$ belong to $L^{m(s)}(G \times J)$, because $D u \in L^{s}(G \times J), u_{t} \in L^{2}(Q)$ and $f \in$ $L^{2}(Q)$. From Lemma 5 again $u_{x_{t} x_{j}} \in L^{k(s)}((G \cap U) \times J)$ for any $(i, j) \neq$ $(n, n)$. Further, let us remark that inequalities $m(s)<k(s) \leqq s$ hold under the conditions of this lemma, see Remark 1 . Thus, the assertion of this lemma is established

q.e.d.

3.

In this section we prove our theorem. We use the lemmas in Section 2 and employ the method of the iteration used in [9] where J.-P. Raymond considered solutions of nonlinear elliptic differetial equations. After it we give some remarks.

Proof of our theorem. Because $D u \in L^{p}(G \times I)$, it follows that $D^{2} u \in$ $L^{m(p)}(G \times I)$ from Lemma 6 and $(D u)_{t} \in L^{k(p)}\left(G \times J_{1}\right)$ from Lemma 5 with $J_{1}=$ $\left(a_{1}, b_{1}\right), a_{1}=a^{\prime} / 2, b_{1}=\left(b^{\prime}+T\right) / 2$, where $0<a^{\prime}<a<b<b^{\prime}<T$.

Therefore, it holds that $D u \in W^{1, m(p)}\left(G \times J_{1}\right)$ from $m(p)<k(p)=p$. Accordingly, by Sobolev's theorem 


$$
\begin{aligned}
D u \in L^{p_{1}}\left(G \times J_{1}\right), p_{1} & =(n+1) m(p) /(n+1-m(p)) \\
& =2(n+1) p /\{(n-1) p+2(n+1)(2-p)\} .
\end{aligned}
$$

Here let us remark that inequalities $p \leqq p_{1}<p_{\infty}$ hold from the assumption (A.1). By virtue of (3.1), Lemma 5 and Remark 4 we conclude that

$$
D^{2} u \in L^{k\left(p_{1}\right)}\left(J_{1}, L_{\mathrm{loc}}^{k\left(p_{1}\right)}(G)\right),(D u)_{t} \in L^{k\left(p_{1}\right)}\left(G \times J_{2}\right),
$$

where $J_{2}=\left(a_{2}, b_{2}\right), a_{2}=\left(a^{\prime}+a_{1}\right) / 2$ and $b_{2}=\left(b^{\prime}+b_{1}\right) / 2$. Moreover, from Lemma 6, (3.1), (3.2) and inequalities $m\left(p_{1}\right)<k\left(p_{1}\right)<p_{1}$

$$
D^{2} u \in L^{m\left(p_{1}\right)}\left(G \times J_{1}\right),(D u)_{t} \in L^{k\left(p_{1}\right)}\left(G \times J_{2}\right) \subset L^{m\left(p_{1}\right)}\left(G \times J_{2}\right) .
$$

Consequently

$$
D u \in W^{1, m\left(p_{1}\right)}\left(G \times J_{2}\right) .
$$

Therefore, by Sobolev's theorem again

$$
D u \in L^{p_{2}}\left(G \times J_{2}\right) .
$$

where

$$
\begin{aligned}
& p_{2}=(n+1) m\left(p_{1}\right) /\left(n+1-m\left(p_{1}\right)\right) \\
= & 2(n+1) p_{1} /\left\{(n-1) p_{1}+2(n+1)(2-p)\right\} .
\end{aligned}
$$

Now let us consider the function $h(s)=2(n+1) s /\{(n-1) s+2(n+1)$. $(2-p)\}$. By simple calculations we see that (i) $h(s)$ is a monotone increasing function of $s, 0 \leqq s$, (ii) if $0<s \leqq p_{\infty}$, then $s \leqq h(s) \leqq h\left(p_{\infty}\right)=p_{\infty}$, (iii) if $2(n+1) /(n+3) \leqq p<2$, then $p \leqq p_{\infty}$ and $p \leqq h(p)=p_{1} \leqq p_{\infty}$.

After repeating processes from (3.1) to (3.5) inductively, we conclude that

$$
D u \in L^{p_{j}}\left(G \times J_{j}\right) . j=3,4, \ldots, .
$$

Here $\left\{p_{j}\right\}_{j=3}^{\infty}$ and $\{J\}_{j=3}^{\infty}$ are defined as follows: for $j=3,4, \ldots$,

$$
\begin{gathered}
p_{j}=2(n+1) p_{j-1} /\left\{(n-1) p_{\jmath-1}+2(2-p)(n+1)\right\}, \\
J_{j}=\left(a_{j}, b_{j}\right), a_{\jmath}=\left(a_{\jmath-1}+a^{\prime}\right) / 2 \text { and } b_{j}=\left(b_{\jmath-1}+b^{\prime}\right) / 2 .
\end{gathered}
$$

From (3.7) and (3.8) it is easy to see that $p_{j} \rightarrow p_{\infty}, a_{l} \rightarrow a^{\prime}$ and $b_{j} \rightarrow b^{\prime}$ as $j \rightarrow \infty$. Then, from (3.6)

$$
D u \in L^{p_{\infty}}\left(G \times I^{\prime \prime}\right), I^{\prime \prime}=\left(a^{\prime}, b^{\prime}\right) .
$$

By virtue of (3.9), Remark 4, Lemmas 5 and 6 it holds that 


$$
\begin{gathered}
D^{2} u \in L^{k\left(p_{\infty}\right)}\left(I^{\prime \prime}, L_{\mathrm{loc}}^{k\left(p_{\infty}\right)}(G)\right),(D u)_{t} \in L^{k\left(p_{\infty}\right)}\left(G \times I^{\prime}\right), \\
D^{2} u \in L^{m\left(p_{\infty}\right)}\left(G \times I^{\prime \prime}\right) .
\end{gathered}
$$

In this way we complete the proof of our theorem.

q.e.d.

Remark 5. (i) In the above proof we see that if $n=1$, then $p_{\infty}=\infty$ and $k\left(p_{\infty}\right)=m\left(p_{\infty}\right)=2$.

(ii) The inequality $p_{1} \leqq p$ holds in the proof of our theorem when the assumption (A.1) fails. In such case we have the following corollary from Remark 4, Lemmas 5 and 6 in place of our theorem:

COROLlary. Let $2 n /(n+2)<p \leqq 2(n+1) /(n+3)$. Under the assumptions (A.2)-(A.4) there exists a unique solution $u$ of (2) such that

(i) $D^{2} u \in L^{p}\left(I, L_{\text {loc }}^{p}(G)\right),(D u)_{t} \in L^{p}\left(G \times I^{\prime}\right)$

(ii) $D^{2} u \in L^{2 p /(4-p)}(Q)$.

Remark 6. By easy calculations we can show that all assertions of our theorem are valid even if we replace the nonlinear operator $A(t)$ by the following nonlinear operator $A_{1}(t)$ with a lower order term:

$$
\left\langle A_{1}(t) v, w\right\rangle=\langle A(t) v, w\rangle+\left(a_{0}(., t, v), w\right) v, w \in V .
$$

Here the function $a_{0}(x, t, z)$ satisfies the assumption

(A.5) $a_{0}(x, t, z) \in C^{0}\left(Q \times R^{1}\right) \cap C^{1}\left(Q \times\left(R^{1}-\{0\}\right)\right)$ and for $(x, t) \in$ $Q, z \in R^{1}-\{0\}$,

$$
\begin{aligned}
& \left|a_{0}(x, t, z)\right| \leqq \Gamma|z|^{\alpha+1} \\
& (\partial / \partial z) a_{0}(x, t, z) \geqq \kappa_{0}|z|^{\alpha}, \\
& \left|(\partial / \partial t) a_{0}(x, t, z)\right|,\left|\left(\partial / \partial x_{k}\right) a_{0}(x, t, z)\right| \leqq \Gamma|z|^{\alpha+1}, k=1, \ldots, n,
\end{aligned}
$$

with some positive constants $\kappa_{0}$ and $\Gamma$, where $0 \leqq \alpha$ if $n=1$, and $0 \leqq \alpha<$ $n p /(n-p)-2$ if $2 \leqq n$. Moreover, if $0 \leqq \alpha<(n+1)(p-1) /(n+1-p)$ -1 , we can replace the inequality $(\partial / \partial z) a_{0}(x, t, z) \geqq \kappa_{0}|z|^{\alpha}$ by the inequality $(\partial / \partial z) a_{0}(x, t, z) \geqq 0$.

\section{REFERENCES}

[1] N. D. Alikakos-L. C. Evans, Continuity of the gradient for weak solutions of a degenerate parabolic equation, J. Math. Pures Appl., 62 (1983), 253-268.

[2] - -R. Rostamian, Gradient estimates for degenerate diffusion equations I, Math. 
Ann., 259 (1982), 53-70.

[ 3 ] S. Campanato, Hölder continuity of the solution of some non-linear elliptic systems, Adv. Math., 48 (1983), 16-43.

[4] F. de Thelin, Local regularity properties for the solutions of a nonlinear partial differential equation, Nonlinear Analy. T. M. A., 6-8 (1982), 839-844.

[5] E. DiBenedetto-A. Friedman, Regularity of solutions of nonlinear degenerate parabolic systems, J. reine angew. Math., 349 (1984), 83-128.

[6] D. Gilbarg-N. S. Trudinger, Elliptic Partial Differential Equations of Second Order, Springer G. M. W. 224, 1977.

[7] H. Nagase, On an application of Roth's method to nonlinear parabolic variational inequalities, Funk. Ekvac., 32-2 (1989), 273-299.

[8] J. Naumann, Interior integral estimates on weak solutions of certain degenerate elliptic systems, Ann. Mat. Pura Appl., (IV), CLVI (1990), 113-125.

[9] J.-P. Raymond, Régularité globale des solutions de systems elliptiques non linéaires, Rev. Mat. Univ. Comp. Madrid, 2-2/3 (1989), 241-270.

[10] S. L. Sobolev, Applications of Functional Analysis in Mathematical Physics, Amer. Math. Soc., Providence, RI, 1963.

[11] P. Tolksdorf, Everywhere-regularity for some quasilinear systems with lack of ellipticity, Ann. Mat. Pura Appl., (4) 134 (1983), 241-266.

Suzuka College of Thechnogy

510-02 Suzuka

Japan 